\title{
ON ASYMPTOTIC OCCUPANCY TIMES OF THE WIENER PROCESS
}

\author{
MALCOLM GOLDMAN
}

1. Introduction. Let $x(t)$ be the one dimensional Wiener process and let $A$ be a measurable subset of the positive reals. We study the classical problem of the asymptotic distribution of the amount of time which the process spends in the set $A$. I.e., if $\chi$ is the characteristic function of the set $A$ we seek the limiting distribution of $T(t)$ $=\int_{0}^{t} \chi(x(u)) d u$ after a suitable normalization. The answer is well known in two cases. (1) $A$ is a half line and the arcsin law is the limiting law and (2) $A$ is a bounded set and the limiting distribution is a Mittag-Leffler distribution. This paper handles the case where $\int_{0}^{x} \chi(u) d u \sim k x^{p}$ for $0 \leqq p \leqq 1$ and $k>0$. The method of Darling and $\mathrm{Kac}$ [1] is the basic tool. Thus the asymptotic behavior of $s \int_{0}^{\infty} e^{-s t}(T(t))^{n} d t$ as $s \rightarrow 0$ determines the $n$th moment of the limiting distribution. Our result is stated in equations (2.4) and (2.5). However, instead of employing an "infinite part" [1, p. 445], we use the Plancherel theorem to obtain the $n$th moment. This leads to an $n$ fold integral which has resisted evaluation except in special cases (the known cases). However, the normalizing function for $T(t)$ is identifiable without evaluation of those integrals.

The technique for handling the above mentioned sets $A$ is described in $\$ 2$. In $\$ 3$, we make a number of additional remarks.

Throughout the paper $E$ is the expectation operator and $\int$ is $\int_{-\infty}^{\infty}$.

The author is indebted to D. A. Darling for pointing out the problem and for a number of conversations on the subject.

2. A special case. Let $A \subset(0, \infty)$ and let $\chi$ be the characteristic function of the set $A$. For $0 \leqq p \leqq 1$ and for $k>0$ we assume

$$
\int_{0}^{x} \chi(u) d u \sim k x^{p} \quad \text { as } \quad x \rightarrow \infty .
$$

If $\psi(z)=\int_{0}^{\infty} e^{-z x} \chi(x) d x$ where $\operatorname{Re} z>0$ it is well known that (2.1) is equivalent to $z^{p} \psi(z) \rightarrow k \Gamma(p+1)$ as $z \rightarrow 0$ through positive reals. Following $[1, \S 2]$ we consider

Presented to the Society, April 22, 1961 under the title Generalized arcsin laws; received by the editors May 29, 1961. 


$$
\begin{aligned}
s \int_{0}^{\infty} E[ & {\left.\left[\int_{0}^{t} \chi(x(u)) d u\right)^{m}\right] e^{-s t} d t=s \int_{0}^{\infty} e^{-s t} \mu_{m}(t) d t } \\
& =(2 s)^{-m / 2} m ! \int \cdots \int \prod_{j=1}^{m} \exp \left(-(2 s)^{1 / 2}\left|x_{j}-x_{j-1}\right|\right) \chi\left(x_{j}\right) d x_{j}
\end{aligned}
$$

where $x_{0}=0$. Now $\chi$ is not integrable so we multiply and divide the $j$ th term of the product in the integrand by $\exp \left(-(2 a s)^{1 / 2} x_{j}\right)$, and obtain

$$
\prod_{j=1}^{m} \exp \left(-(2 s)^{1 / 2}\left|x_{j}-x_{j-1}\right|+(2 a s)^{1 / 2} x_{j}\right) \chi\left(x_{j}\right) \exp \left(-(2 a s)^{1 / 2} x_{j}\right)
$$

If $a<m^{-2}$ repeated integration shows that

$$
\prod_{j=1}^{m} \exp \left(-(2 s)^{1 / 2}\left|x_{j}-x_{j-1}\right|+(2 a s)^{1 / 2} x_{j}\right) \in L_{2}\left(E_{m}\right) .
$$

To obtain a more useful form we let $a^{1 / 2}=b$ and use the Plancherel theorem. This is justified since $A \subset(0, \infty)$ so both factors in (2.2) are $L_{2}$ functions when $a<m^{-2}$. If we let $y_{j}=x_{1}+\cdots+x_{j}$ we get a transformation with unit Jacobian with $x_{j}-x_{j-1}=y_{j}$ and the Fourier transform of $(2.3)$ is

$$
\begin{aligned}
& \int \cdots \int \prod_{j=1}^{m} \\
& \quad \cdot \exp \left[i\left(\alpha_{1}+\cdots+\alpha_{j}-j i(2 s)^{1 / 2} b\right) y_{j}+(2 s)^{1 / 2}\left|y_{j}\right|\right] d y_{1} \cdots d y_{m}
\end{aligned}
$$

which is absolutely convergent for each $s>0$ when $b<m^{-1}$. Now since $\int e^{b y-c|y|} d y=2 c\left(c^{2}-b^{2}\right)^{-1}$ if $0<\operatorname{Re} b<c$ we obtain

$$
\begin{aligned}
& s \int_{0}^{\infty} \mu_{m}(t) e^{-s t} d t \\
& \quad=m ! \pi^{-m} \int \cdots \int \prod_{j=1}^{m} \frac{\psi\left((2 s)^{1 / 2} b+i \alpha_{j}\right) d \alpha_{j}}{\left[\left(\alpha_{1}+\cdots+\alpha_{j}-j i(2 s)^{1 / 2} b\right)^{2}+2 s\right]} .
\end{aligned}
$$

Replacing $\alpha_{j}$ by $(2 s)^{1 / 2} \alpha_{j}$ yields

$$
m ! \pi^{-m}(2 s)^{-m / 2} \int \cdots \int \prod_{j=1}^{m} \frac{\psi\left((2 s)^{1 / 2}\left(b+i \alpha_{j}\right)\right) d \alpha_{j}}{\left[\left(\alpha_{1}+\cdots+\alpha_{j}-j i b\right)^{2}+1\right]} .
$$

By selecting a proper branch of the logarithm function we may arrange that $z^{p} \psi(z)$ is analytic if $\operatorname{Re} z>0$, thus by Abel's theorem and by $(2.1)$ 


$$
\left.\lim _{s \rightarrow 0^{+}}\left((2 s)^{1 / 2}\left(b+i \alpha_{j}\right)\right)\right)^{p} \psi\left((2 s)^{1 / 2}\left(b+i \alpha_{j}\right)\right)=k \Gamma(p+1) .
$$

If $r=\operatorname{Re} z,\left|\int_{0}^{\infty} e^{-z x} \chi(x) d x\right| \leqq \int_{0}^{\infty} e^{-r x} \chi(x) d x$ so for fixed $b<m^{-1}$

$$
\begin{aligned}
\prod_{j=1}^{m} \psi\left((2 s)^{1 / 2}(b+\right. & \left.\left.i \alpha_{j}\right)\right)\left[\left(\alpha_{1}+\cdots+\alpha_{j}-j i b\right)^{2}+1\right]^{-1} \\
& =(2 s)^{-m p / 2} O\left(\prod_{j=1}^{m}\left[\left(\alpha_{1}+\cdots+\alpha_{j}\right)^{2}+1-m^{2} b^{2}\right]^{-1}\right) .
\end{aligned}
$$

The substitution $\beta_{j}=\alpha_{1}+\cdots+\alpha_{j}$ shows that the last function is integrable on $E_{m}$ and since it is independent of $s$ the dominated convergence theorem implies that (2.4) is asymptotic to

$m ! \pi^{-m}(k \Gamma(p+1))^{m}(2 s)^{-m(1+p) / 2}$

$$
\cdot \int \cdots \int \prod_{j=1}^{m} \frac{d \alpha_{j}}{\left(b+i \alpha_{j}\right)^{p}\left[\left(\alpha_{1}+\cdots+\alpha_{j}-j i b\right)^{2}+1\right]} \cdot
$$

We denote the last integral by $(2 \pi)^{m} I_{m}$ and have

$$
s \int_{0}^{\infty} \mu_{m}(t) e^{-s t} d t \sim m !\left(2 k \Gamma(p+1)(2 s)^{-(1+p) / 2}\right)^{m} I_{m} .
$$

If we set $c=2^{-(p-1) / 2} k \Gamma(p+1)$ since $\mu_{m}(0)=0$, Karamata's Tauberian theorem implies that

$$
\begin{aligned}
E\left(\left[c^{-1} t^{-(1+p) / 2} \int_{0}^{t} \chi(x(u)) d u\right]^{m}\right) & \rightarrow \frac{m !}{\Gamma\left(\frac{1}{2}(1+p) m+1\right)} I_{m} \\
& =\mu_{m} .
\end{aligned}
$$

Now $\mu_{m}=\lim _{t \rightarrow \infty} c^{-1} t^{-(1+p) / 2} \mu_{m}(t)$ and since the condition for positive definiteness of a sequence $\left\{\mu_{m}\right\}$ depends on only finitely many terms at each stage, it is clear that our sequence $\left\{\mu_{m}\right\}$ is the moment sequence of some probability measure $P$ on $(0, \infty)$. Now $I_{m}$ does not grow too fast. Indeed since $I_{m}$ does not depend on $b$ we may choose $b$ so small that $\left|\left(\alpha_{1}+\cdots+\alpha_{j}-j i b\right)^{2}+1\right| \geqq 1-j^{2} b^{2}+\beta_{j}^{2}>1 / 2+\beta_{j}^{2}$. We therefore majorize the typical term in our product by $2\left|\alpha_{j}\right|^{-p}$ if $\left|\alpha_{j}\right| \leqq 1$ and by $\left(1 / 2+\beta_{j}^{2}\right)^{-1}$ if $\left|\alpha_{j}\right|>1$. Hence there is a constant $l$ such that $\left|I_{m}\right| \leqq l^{m}$ and $\sum_{m=0}^{\infty}\left(\mu_{m} / m !\right) z^{m}$ is an entire function. Thus there is only one probability measure having the moment sequence $\left\{\mu_{m}\right\}$ and by Helly's theorem as $t \rightarrow \infty$, the distribution of $c^{-1} t^{-(1+p) / 2} T(t)$ approaches $P$ in distribution.

We next show that $P$ is nontrivial. This is equivalent to the inequality 


$$
2\left(\Gamma\left(\frac{1}{2} p+\frac{3}{2}\right)\right)^{2} I_{2}>\Gamma(2+p) I_{1}^{2} .
$$

We assume $p \neq 1$ since in that case one obtains the moments of the arcsin law. Using the branch of the logarithm on which $z^{p}=|z|{ }^{p} e^{i p \text { arg } z}$ with $-\pi<\arg z<\pi, I_{1}$ can be evaluated by contour integration on the semi-circle in the lower half-plane with diametral segment $[-M, M]$. There is a simple pole in the interior at the point $-i+i b$. Since the integrand grows like $\left|\alpha_{1}\right|^{-2-p}$, the contribution of the circular part approaches zero and

$$
I_{1}=(2 \pi)^{-1} \int_{-\infty}^{\infty} \frac{d \alpha}{(b+i \alpha)^{p}\left[(\alpha-i b)^{2}+1\right]}=-i(-2 i)^{-1}=\frac{1}{2} .
$$

The same contour integration applied to $I_{2}$ together with the dominated convergence theorem gives

$$
\begin{aligned}
I_{2} & =(-2 \pi)^{-1} i \int_{-\infty}^{\infty}[b+i(-i-\beta+2 i b)]^{-p}[-2 i]^{-1}\left[(\beta-i b)^{2}+1\right]^{-1} \\
& \cdot[b+i \beta]^{-p} d \beta \\
& =(4 \pi)^{-1} \int_{-\infty}^{\infty}(1-i \beta-b)^{-p}\left[(\beta-i b)^{2}+1\right]^{-1}[b+i \beta]^{-p} d \beta .
\end{aligned}
$$

$z^{p}$ has been chosen so that $\left(z^{*}\right)^{p}=\left(z^{p}\right)^{*}$ and since $\int_{0}^{e}|\beta|^{-p} d \beta<\infty$, the last integral can be written, after letting $b \rightarrow 0$, as

$$
\begin{aligned}
(4 \pi)^{-1} & \int_{-\infty}^{\infty}\left(\beta^{2}+1\right)^{-1-p / 2}|\beta|^{-p} \exp \left(-i p\left(\frac{\pi}{2} \operatorname{sg} \beta-\arctan \beta\right)\right) d \beta \\
& =(2 \pi)^{-1} \int_{0}^{\infty}\left(\beta^{2}+1\right)^{-1-1 / 2}|\beta|^{-p} \cos p(\operatorname{arccot} \beta) d \beta \\
& =(2 \pi)^{-1} \int_{0}^{\pi / 2} \cos ^{-p} w \sin ^{2 p} w \cos p w d w
\end{aligned}
$$

which we call $b_{p}$. Hence the problem is that of showing that for $0 \leqq p<1$

$$
8\left(\Gamma\left(\frac{1}{2} p+\frac{3}{2}\right)\right)^{2} b_{p}>\Gamma(2+p) .
$$

It was necessary to subdivide the interval $(0,1)$ into a number of pieces and to resort to numerical methods to estimate $b_{p}$ but the inequality is valid. It is immediate that equality can occur for at most finitely many $p \in(0,1)$ since the terms in $(2.6)$ are analytic in a region containing the open interval $(0,1)$ and $\lim _{p \rightarrow 0} b_{p}=1$ while $b_{p}$ is greater than a function whose limit as $p \rightarrow 1$ is $8^{-1}+(2 \pi)^{-1}$. 
3. Concluding remarks. The method of $\$ 2$ applies to more cases than those directly indicated. In fact, if $A$ is such that $\int_{0}^{x} \chi(u) d u$ $\sim k \log x$ the limit distribution of $(c t \log t)^{-1} T(t)$ is the Mittag-Leffler distribution of index $1 / 2$ (compare with the case of a bounded set [1]) if $c$ is suitably chosen. Also in view of $[2$, p. 85] it would be informative to ascertain the limiting distribution when

$$
A=\left\{x \mid 2^{2 n} \leqq x \leqq 2^{2 n+1}, n=0,1,2, \cdots\right\} .
$$

It should also be possible to apply the method to occupancy of the "first octant" by multi-dimensional Wiener processes.

In order to apply this technique to processes other than the Wiener process it would be desirable to use functions of slower growth than the exponential function to obtain an expression akin to (2.2).

Lastly, if one sets $I_{0}(z)=1$ and

$$
I_{m+1}(z)=(2 \pi)^{-1} \int \frac{I_{m}(\alpha) d \alpha}{\left[(\alpha+z-i b)^{2}+1\right][b+i \alpha]^{p}},
$$

then $I_{m}(0)=I_{m}$. Letting $\phi(z)=\sum_{m=0} \lambda^{m} I_{m}(z)$, one obtains, formally,

$$
\phi(z)=1+\lambda(2 \pi)^{-1} \int \frac{\phi(\alpha) d \alpha}{\left[(\alpha+z-i b)^{2}+1\right][b+i \alpha]^{p}} .
$$

It can be arranged for $\phi$ to be bounded in any half-plane Im $z \geqq y$ where $y>-1$. Solution of this equation would give the moments of the limiting distribution in view of (2.5).

\section{REFERENCES}

1. D. A. Darling and M. Kac, On occupation times for Markoff processes, Trans. Amer. Math. Soc. 84 (1957), 444-458.

2. G. H. Hardy, Divergent series, Oxford Univ. Press, Oxford, 1956.

UNIVERSITY OF MICHIGAN AND ReEd College 